\title{
DOA estimation of wideband signals based on iterative spectral reconstruction
}

\author{
Shun $\mathrm{He}^{1,2}$, Zhiwei Yang ${ }^{2, *}$, and Guisheng Liao ${ }^{2}$ \\ 1. National Laboratory of Radar Signal Processing, Xidian University, Xi' an 710071, China; \\ 2. Communication and Information Engineering Collage, Xi' an University of Science and Technology, Xi' an 710054, China
}

\begin{abstract}
In order to solve the problem of coherent signal subspace method (CSSM) depending on the estimated accuracy of signal subspace, a new direction of arrival (DOA) estimation method of wideband source, which is based on iterative adaptive spectral reconstruction, is proposed. Firstly, the wideband signals are divided into several narrowband signals of different frequency bins by discrete Fourier transformation (DFT). Then, the signal matched power spectrum in referenced frequency bins is computed, which can form the initial covariance matrix. Finally, the linear restrained minimum variance spectral (Capon spectral) of signals in other frequency bins are reconstructed using sequential iterative means, so the DOA can be estimated by the locations of spectral peaks. Theoretical analysis and simulation results show the proposed method based on the iterative spectral reconstruction for the covariance matrices of all sub-bands can avoid the problem of determining the signal subspace accurately with the coherent signal subspace method under the conditions of small samples and low signal to noise ratio (SNR), and it can also realize full dimensional focusing of different sub-band data, which can be applied to coherent sources and can significantly improve the accuracy of DOA estimation.
\end{abstract}

Keywords: direction of arrival (DOA), wideband source, coherent source, minimum variance spectral.

DOI: $10.21629 / J S E E .2017 .06 .01$

\section{Introduction}

Direction of arrival (DOA) estimation for wideband source is a research hotspot in array signal processing, and it is widely used in radar, sonar, communication, and so on $[1-3]$. The envelope of the wideband signal changes rapidly, so in the far-field plane wave assumption, the received signals of array sensors have not only the envelope

\footnotetext{
Manuscript received November 19, 2015.

*Corresponding author.

This work was supported by the National Natural Science Foundation of China (61671352), the open foundation of Key Laboratory of Cognitive Radio and Information Processing, Ministry of Education (Guilin University of Electronic Technology) (CRKL160206), and Xi' an University of Science and Technology Doctor (after) Start Gold Project (2017QDJ018).
}

delay, but also the phase change, and the phase change is not only dependent on the DOA of sources, but also related to the frequency of the sources, so the traditional DOA estimation methods for narrowband signals are no longer suitable for wideband signals.

DOA estimation for the wideband source, in strict sense, requires envelope alignment by delay units. However, in order to avoid the operation of envelop alignment in engineering, the subband signals (each subband corresponds to a narrow band signal) formed by discrete Fourier transform (DFT) are usually used to estimate the DOA of wideband sources. The incoherent signal subspace method (ISSM) and the coherent signal subspace method (CSSM) are the typical DOA estimation algorithms of wideband sources. The output of array sensors are partitioned into non-overlapping narrowband signals by the sub-band processing. Each one of these narrowband signals is processed individually, and the results are averaged to get the DOA of wideband signals [4,5]. This approach is referred to as the ISSM. It is simple, but requires a high signal to noise ratio (SNR) and a large training sample set, and it cannot directly deal with the coherent sources. The CSSM can handle coherent sources, where the covariance matrices are estimated at several frequency bins, aligned by prober transformation matrices (for example rotational signal subspace (RSS) [6], signal subspace transformation (SST) [7], unitary constrained array manifold (UCAM) [8], two sided correlation transformation (TCT) [9] and modified total least-square (MTLS) [10]), and finally averaged. Compared to the ISSM algorithm, the CSSM algorithm significantly extends the detection and resolution thresholds, and also improves the bias and accuracy performance at a low SNR, and it is further able to cope with coherent sources in multipath scenarios. The CSSM requires initial values to find focusing matrices, and the estimation performance of CSSM is sensitive to these initial values. In fact, poor initial values can lead to biased 
estimates. In [11], a DOA estimation algorithm for wideband sources called test orthogonality of projected subspaces (TOPS) has been proposed, and it does not require any preprocessing for initial values, but when there are multiple signals it is prone to pseudo peaks. Based on the sparse signal processing theory, a new solution of DOA estimation for wideband sources has been given in [12-15]. In addition, Wu et al has proposed a DOA estimation method of wideband sources using the particle filter [16], which can avoid the problem of estimating the covariance matrix, but that has the problems of high computational complexity and particle degradation.

The ISSM and CSSM need to estimate the data covariance matrices of non-overlapping narrowband signals, however, under the condition of the small samples and the low SNR, the estimation error of covariance matrices can lead to distortion of the signal subspace, which will worsen the performance of DOA estimation. Using certain relationship of transformation of the array steer vectors between different frequency bins, a new DOA estimation method for wideband sources is proposed. Firstly, the wideband source is divided into several narrowband signals by DFT. Then, the signal matched power spectral in referenced frequency bins is computed, which forms the initial covariance matrix. Finally, the linear restrained minimum variance spectral (Capon spectral) of signals in other frequency bins are reconstructed using sequential iterative means, the DOA can be estimated by locations of spectral peaks. Theoretical analysis and simulation results show that the proposed method can significantly improve the estimation accuracy of the DOA under the small snapshots and the low SNR, and it is suitable for coherent source signals.

\section{Signal model}

Consider a uniform linear array composed of $M$ identical sensors separated from each other by a distance $d=\lambda / 2$, where $\lambda$ is the wavelength corresponding to the highest temporal frequency component of the received signals. Let the $K$ wideband sources impinge on the array from directions $\left\{\theta_{1}, \ldots, \theta_{K}\right\}$ with an overlapping spectrum of the bandwidth. Then, the received signal at the $m$ th sensor can be expressed as

$$
\begin{gathered}
x_{m}(t)=\sum_{k=1}^{K} s_{k}\left(t-\tau_{m}\left(\theta_{k}\right)\right)+n_{m}(t), \\
m=1,2, \ldots, M
\end{gathered}
$$

where $s_{k}(t)$ is the $k$ th signal source, $\theta_{k}$ is the angle of arrival for the $k$ th source, $\tau_{m}\left(\theta_{k}\right)$ is the propagation delay for the $k$ th source at the $m$ th sensor with respect to the reference point of the array, and $n_{m}(t)$ is additive noise at the $m$ th sensor. Suppose that the observation time is long enough so that the Fourier transform of the sensor output has good resolution. The discrete-time Fourier transform (DTFT) of the $m$ th sensor output is

$$
\begin{gathered}
X_{m}\left(f_{i}\right)=\sum_{k=1}^{K} \mathrm{e}^{-\mathrm{j} 2 \pi f_{i} \tau_{m}\left(\theta_{k}\right)} S_{k}\left(f_{i}\right)+N_{m}\left(f_{i}\right), \\
m=1,2, \ldots, M ; i=1,2, \ldots, J
\end{gathered}
$$

where $S_{k}\left(f_{i}\right)$ and $N_{m}\left(f_{i}\right)$ are the discrete Fourier coefficient of $s_{k}(t)$ and $n_{m}(t)$ at frequency $f_{i}$, respectively. $J$ is the number of frequency division in the bandwidth $\left[f_{L}, f_{H}\right]$. Formula (2) can be written in vector form, as follows:

$$
\boldsymbol{X}\left(f_{i}\right)=\boldsymbol{A}\left(f_{i}, \boldsymbol{\theta}\right) \boldsymbol{S}\left(f_{i}\right)+\boldsymbol{N}\left(f_{i}\right)
$$

where $\boldsymbol{X}\left(f_{i}\right)=\left[X_{1}\left(f_{i}\right), \ldots, X_{M}\left(f_{i}\right)\right]^{\mathrm{T}}$ is the receive data vector, $\boldsymbol{S}\left(f_{i}\right)=\left[S_{1}\left(f_{i}\right), \ldots, S_{K}\left(f_{i}\right)\right]^{\mathrm{T}}$ is the signal vector, $\boldsymbol{N}\left(f_{i}\right)=\left[N_{1}\left(f_{i}\right), \ldots, N_{M}\left(f_{i}\right)\right]^{\mathrm{T}}$ is the noise vector, $\boldsymbol{A}\left(f_{i}, \boldsymbol{\theta}\right)=\left[\boldsymbol{a}\left(f_{i}, \theta_{1}\right), \boldsymbol{a}\left(f_{i}, \theta_{2}\right), \ldots, \boldsymbol{a}\left(f_{i}, \theta_{K}\right)\right]$ is the $M \times K$ array manifold matrix, $\boldsymbol{a}\left(f_{i}, \theta_{k}\right)=$ $\left[\mathrm{e}^{-\mathrm{j} 2 \pi f_{i} \tau_{1}\left(\theta_{k}\right)}, \mathrm{e}^{-\mathrm{j} 2 \pi f_{i} \tau_{2}\left(\theta_{k}\right)}, \ldots, \mathrm{e}^{-\mathrm{j} 2 \pi f_{i} \tau_{M}\left(\theta_{k}\right)}\right]^{\mathrm{T}}$ is the steering vector, $\tau_{m}\left(\theta_{k}\right)=\frac{(m-1) d \sin \theta_{k}}{c}$ and $c$ is the propagation velocity.

Obviously, the array manifold matrix $\boldsymbol{A}\left(f_{i}, \boldsymbol{\theta}\right)$ of wideband sources is related to the frequency, the fusion processing (such as the ISSM method) of DOA estimation at each frequency bin does not make use of certain relationship of transformation of array manifold matrices at different frequency bins. The CSSM class method transforms the $\boldsymbol{A}\left(f_{i}, \boldsymbol{\theta}\right)$ at different frequency bins into signal subspace of reference frequency bins by using the focusing matrix, then a high-resolution method such as the multiple signal classification (MUSIC) is used to find the DOA. However, the estimation accuracy of the CSSM class method is related to the estimation accuracy of $\boldsymbol{A}\left(f_{i}, \boldsymbol{\theta}\right)$, moreover, the focusing process has the mismatch error of the signal subspace model, so the accuracy of DOA estimation will decrease obviously in the small samples and the low SNR.

\section{Iterative adaptive spectral reconstruction}

\subsection{Algorithm description}

The array manifold matrix of wideband sources is related to the frequency, but the DOA of sources does not vary with the frequency, that is to say, the sub-band signals at different frequency bins have identical spatial spectrums. Thus, we can estimate the DOA of sources by direct "focusing" spatial spectrum of different frequency bins, which can avoid the problem of determining the dimension of signal subspace and realize the full dimensional focusing for wideband sources. 
The matched beam-forming or the linear constrained minimum variance criterion can be used to estimate the spatial spectrum (The former is called matched spectrum, the latter is called Capon spectrum). It is well know that the Capon spectrum has the ability to distinguish the adjacent signals located in the main lobe of the matched beamforming, so super-resolution of DOA in the main lobe can be realized by focusing the Capon spectrum of the different frequency bins.

The key calculating of the Capon spectrum is to estimate the covariance matrix of received data, which requires a lot of independent identically distributed samples. In fact, when the training samples are adequate and the DOA of sources does not vary with the change of time and the noise is Gaussian white noise, the ideal covariance matrix $\boldsymbol{R}_{I}\left(f_{i}\right)$ of array data at frequency $f_{i}$ can be expressed as

$$
\boldsymbol{R}_{I}\left(f_{i}\right)=\int_{-\pi / 2}^{\pi / 2} \sigma^{2}\left(f_{i}, \theta\right) \boldsymbol{a}\left(f_{i}, \theta\right) \boldsymbol{a}^{\mathrm{H}}\left(f_{i}, \theta\right) \mathrm{d} \theta
$$

where $\boldsymbol{a}\left(f_{i}, \theta\right)$ and $\sigma^{2}\left(f_{i}, \theta\right)$ are the array steering vector and the signal energy, respectively. According to the linear constraint minimum mean square error criterion, the estimation of energy $\sigma^{2}\left(f_{i}, \theta\right)$ can be given by

$$
\widehat{\sigma}^{2}\left(f_{i}, \theta\right)=\boldsymbol{w}^{\mathrm{H}}\left(f_{i}, \theta\right) \boldsymbol{R}_{I}\left(f_{i}\right) \boldsymbol{w}\left(f_{i}, \theta\right)
$$

where $\boldsymbol{w}\left(f_{i}, \theta\right)=\frac{\boldsymbol{R}_{I}^{-1}\left(f_{i}\right) \boldsymbol{a}\left(f_{i}, \theta\right)}{\boldsymbol{a}^{\mathrm{H}}\left(f_{i}, \theta\right) \boldsymbol{R}_{I}^{-1}\left(f_{i}\right) \boldsymbol{a}\left(f_{i}, \theta\right)}$ is the adaptive weighted vector at frequency $f_{i}$ and search angle $\theta$.

In the engineering application, such as in some large scale array or space time adaptive processing and other applications, the number of training samples is often limited, and the ideal covariance matrix is unknown, so we use covariance matrices of all frequency bins to update the energy of the search angle sequentially, and finally, the DOA can be estimated by the locations of spectral peaks. The concrete steps are as follows:

Step 1 Initializing the auto-correlative matrices of array data at all frequency bins: $\widehat{\boldsymbol{R}}\left(f_{i}\right)=\boldsymbol{X}\left(f_{i}\right)$. $\boldsymbol{X}^{\mathrm{H}}\left(f_{i}\right) / L(i=1, \ldots, J)$, where $L$ is the number of snapshots at each frequency bin.

Step 2 Calculating the matched spatial spectrum of the reference frequency $f_{i}$ (Let $i=1$ ): $\hat{\boldsymbol{P}}_{k}\left(f_{i}\right)=$ $\boldsymbol{a}^{\mathrm{H}}\left(f_{i}, \theta_{k}\right) \widehat{\boldsymbol{R}}\left(f_{i}\right) \boldsymbol{a}\left(f_{i}, \theta_{k}\right)$, where $\widehat{\boldsymbol{P}}_{k}\left(f_{i}\right)$ is the power of the $k$ th search angle, $\theta_{k} \in\left[\begin{array}{ll}-\frac{\pi}{2} & \frac{\pi}{2}\end{array}\right]$.

Step 3 Reconstructing the auto-correlative matrix of the $(i+1)$ th frequency bin based on the spatial spectrum of the $i$ th frequency bin:

$$
\widehat{\boldsymbol{R}}_{\boldsymbol{r}}\left(f_{i+1}\right)=\sum_{\theta_{k}=-\pi / 2}^{\pi / 2} \hat{\boldsymbol{P}}_{k}\left(f_{i}\right) \boldsymbol{a}\left(f_{i+1}, \theta_{k}\right) \boldsymbol{a}^{\mathrm{H}}\left(f_{i+1}, \theta_{k}\right) .
$$

Step 4 Updating the spatial spectrum of the $(i+1)$ th frequency bin by the linear constrained minimum variance criterion:

$$
\widehat{\boldsymbol{P}}_{k}\left(f_{i+1}\right)=\boldsymbol{w}^{\mathrm{H}}\left(f_{i+1}, \theta_{k}\right) \hat{\boldsymbol{R}}\left(f_{i+1}\right) \boldsymbol{w}\left(f_{i+1}, \theta_{k}\right)
$$

where $\boldsymbol{w}\left(f_{i+1}, \theta_{k}\right)=\frac{\widehat{\boldsymbol{R}}_{\boldsymbol{r}}^{-1}\left(f_{i+1}\right) \boldsymbol{a}\left(f_{i+1}, \theta_{k}\right)}{\boldsymbol{a}^{\mathrm{H}}\left(f_{i+1}, \theta_{k}\right) \widehat{\boldsymbol{R}}_{\boldsymbol{r}}^{-1}\left(f_{i+1}\right) \boldsymbol{a}\left(f_{i+1}, \theta_{k}\right)}$

Step $5 i=i+1$, going to Step 3, repeating the above process until the spatial spectra of all frequency bins are updated.

Step 6 Getting the DOA estimation of wideband sources by peak locations of spatial spectra.

Obviously, the proposed method not only avoids the problem of determining signal subspace in the small snapshots and the low SNR, but also makes full use of the transformation characteristics of the array manifold at different frequency bins, the full dimensional focusing of the spatial spectra is realized by the sequential iteration, which is expected to achieve better performance of DOA estimation than the coherent signal subspace method.

\subsection{Discussion}

\subsubsection{Small snapshots}

When the number of training samples is small, the covariance matrix of array data will have a large error, which not only leads to distortion of signal subspace obtained by the eigenvalue decomposition, deteriorates the performance of DOA estimation of the subspace class method, but also makes the Capon spatial spectrum have lager disturbance, which reduces the angle resolution. In this paper, the estimation of the Capon spatial spectrum $\widehat{\boldsymbol{P}}_{k}\left(f_{i+1}\right)$ at the $(i+1)$ th frequency bin can be obtained by reconstruction of the covariance matrix at the $(i+1)$ th frequency bin, and the auto-correlative matrix at the $(i+1)$ th frequency bin is obtained by the sum of spatial spectrum estimated value $\hat{\boldsymbol{P}}_{k}\left(f_{i}\right)$ in the interval of $\left[\begin{array}{ll}-\frac{\pi}{2} & \frac{\pi}{2}\end{array}\right]$ at the $i$ th frequency bin. Let the signal sources have the characteristic of flat spectrum, i.e. $\boldsymbol{P}_{k}\left(f_{i}\right)=\boldsymbol{P}_{k}\left(f_{i+1}\right)$, then the reconstructed auto-correlative matrix can be close to the ideal auto-correlative matrix, which can ensure that $\widehat{\boldsymbol{P}}_{k}\left(f_{i+1}\right)$ converges and is the minimum mean square spectrum at the $(i+1)$ th frequency bin. The subsequent simulation shows that the proposed method can improve the detection probability and the estimated accuracy of adjacent signals in small samples.

\subsubsection{Coherent signal}

The number of main eigenvectors, i.e. the eigenvectors corresponding to lager eigenvalues, will decrease for the coherent signal, and the main eigen-space spanned by main eigenvectors is not equivalent to the real array manifold of received data. Thus, not only the DOA estimation method based on focusing of signal subspace will fail, but also the 
minimum mean square spectrum computed by the sample covariance matrix inverse algorithm will distort, which cannot correctly estimate the DOA of coherent signals. The proposed method does not have the problem of the dimension reduction of the main egien-space when the array covariance matrix is reconstructed, therefore, for the coherent signals, the proposed method can get the reconstructed covariance matrix, and the minimum mean square spectrum updated by iterative adaptive processing can be used to realize super resolution of the coherent signals.

\subsubsection{Computational complexity}

In order to analyze the computational complexity of the proposed method, we first discuss the determination of the search interval. The reconstruction of spatial spectrums needs to estimate the search powers at different frequency bins, so increasing the search interval can improve the efficiency of operation, but the accuracy of the DOA estimation is poor; in contrary, it can improve the accuracy of the DOA estimation, but the amount of computation increases. In engineering, the interval of search angles $\Delta \theta$ can be set according to the Cramer-Rao lower bound of the parameter estimation.

Once the interval of search angles is determined, the total number of search angles in the range of $[-\pi / 2, \pi / 2]$ is $Q=\lfloor\pi / \Delta \theta\rfloor$ (where $\lfloor\cdot\rfloor$ is rounding down). Let the number of snapshots at each frequency be $N$, then the computational complexity of the data correlative matrix is $O\left(M^{2} N\right)$, the reconstruction of the correlative matrix is $O\left(M^{2} Q\right)$, the matrix inversion is $O\left(M^{3}\right)$, the spatial spectrum is updated as $O\left(\left(M^{2}+M\right) Q\right)$. The proposed method needs to orderly reconstruct and update the correlative matrix and spatial spectra of $J$ frequency bins, so the total computational complexity is $O\left(J\left(M^{3}+2 M^{2} Q+M^{2} N+\right.\right.$ $M Q))$.

For the coherent subspace method TCT, let the number of initial angles be $K^{\prime}$, then the computational complexity of the data correlative matrix is $O\left(M^{2} N\right)$, the eigenvalue decomposition (computing the signal subspace) is $O\left(M^{3}\right)$, the focusing transformation is $O\left(M^{2} K^{\prime}+2 M^{3}\right)$, the search of peaks is $O(K(M+1) Q)$, so the total computational complexity is $O\left(J\left(M^{3}+M^{2} N\right)+(J-1)\left(M^{2} K^{\prime}+\right.\right.$ $\left.\left.2 M^{3}\right)+K(M+1) Q\right)$.

The interval of search angles is usually small, so the computational complexity of the proposed method is higher than that of the traditional focusing method TCT. However, this method does not need to determine the dimension of signal subspace and estimate the initial value of DOA, it can be used in the coherent source environment, and the estimation performance is better than the traditional focusing method under the conditions of the small snapshots and low SNR. In the engineering application, the parallel processing based on the graphic processing unit (GPU) can effectively improve the speed of operation and realize real-time processing, so the proposed method is available in engineering field.

\section{Simulation results}

In the following simulations, considering a linear array of $M=10$ omnidirectional sensors with equal inter-element spacing $d=\frac{c}{2 f_{0}}$, where $f_{0}$ is the mid-band frequency and $c$ is the velocity of propagation. The two equal power coherent wideband sources are placed at $10^{\circ}$ and $30^{\circ}$ with the same processing bandwidth of $0.8-1.2 \mathrm{GHz}$, and the sensor output is divided into 16 sub-bands $(J=16)$.

Fig. 1 gives the normalized spatial spectrum based on the TCT method and the proposed method (that is called ITER_SPEC) with different SNRs and snapshots. The results show that with higher SNR and more snapshots, the TCT method and the ITER_SPEC method can effectively estimate the DOA of sources; with higher SNR and fewer snapshots, or lower SNR and fewer snapshots, the TCT method is unable to form the correct spectral peaks; instead, the ITER_SPEC can still form the correct spectral peaks at the positions of real DOA.

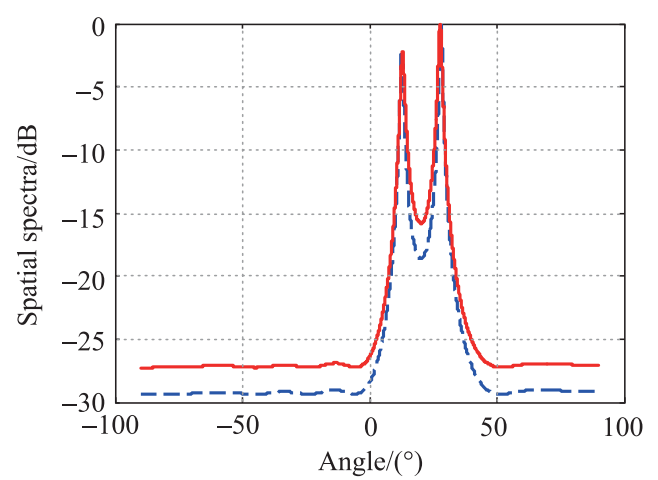

(a) Normalized spatial spectrum when $\mathrm{SNR}=10 \mathrm{~dB}$ and with 30 snapshots

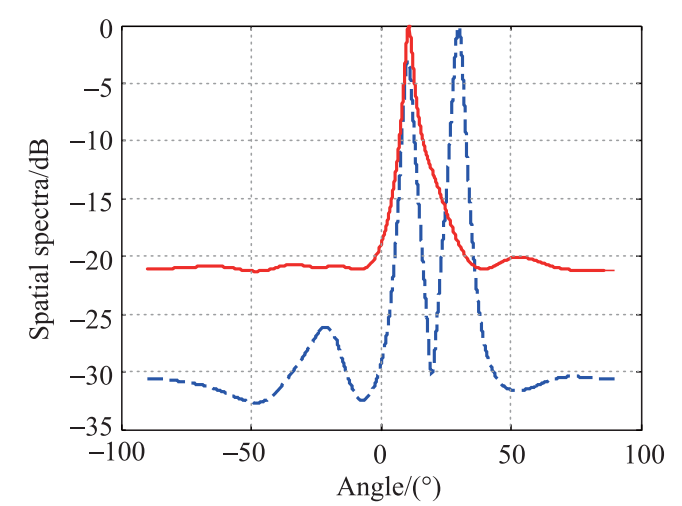

(b) Normalized spatial spectrum when $\mathrm{SNR}=20 \mathrm{~dB}$ and with two snapshots 


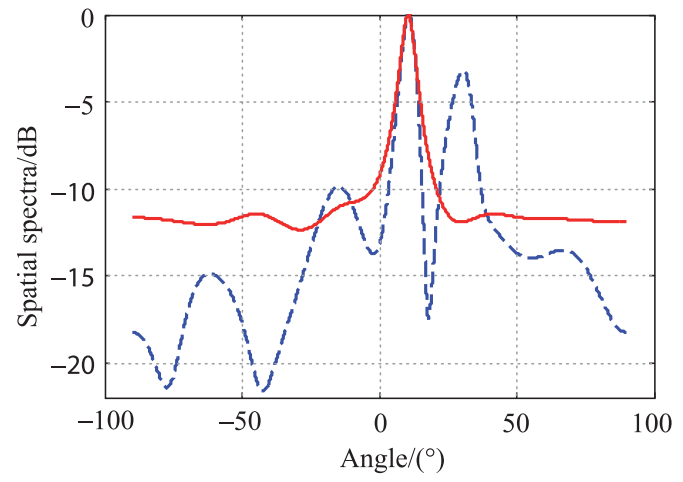

(c) Normalized spatial spectrum when $\mathrm{SNR}=5 \mathrm{~dB}$ and with two snapshots

$$
\text { - - - : ITER_SPEC; }
$$

Fig. 1 Normalized spatial spectrum under different SNRs and snapshots

Fig. 2 shows that the performances of DOA estimation for the TCT method and ITER_SPEC method with the different snapshots. Fig. 2(a) gives the detected probability of two signals, Fig. 2(b) gives the root mean square error (RMSE) of the angle estimate, where Monte-Carlo experiments are 1000 , and the SNR is $10 \mathrm{~dB}$. The definition of the detection probability is the total number divided by the number of successful estimates (if the estimate is within $\pm 1^{\circ}$ of the real value, it is considered to be successful estimate), the definition of RMSE is RMSE = $\left[\sum_{k=1}^{K}\left(\theta_{k}-\hat{\theta}_{k}\right)^{2}\right] / N_{P}$, where $\theta_{k}$ is the real signal DOA, $\hat{\theta}_{k}$ is the DOA estimation value, and $N_{P}$ is the number of successful estimates. It is clear that the performance of the spectral reconstruction method is superior to that of the TCT method, the performance of the TCT is poor under the small samples, but the spectral reconstruction method can correctly estimate the DOA of signals when the number of the snapshots is two.

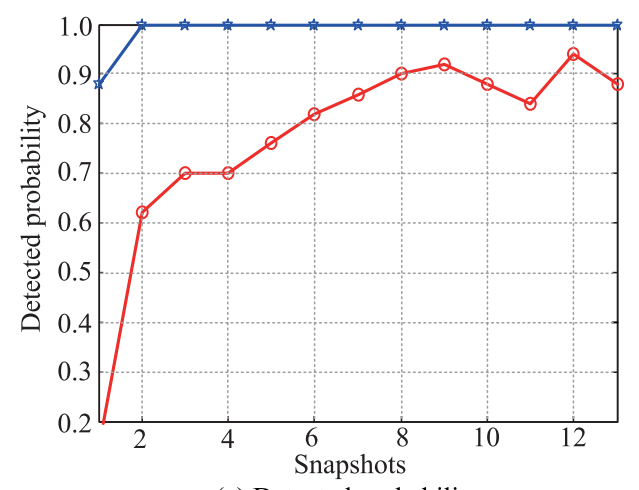

(a) Detected probability

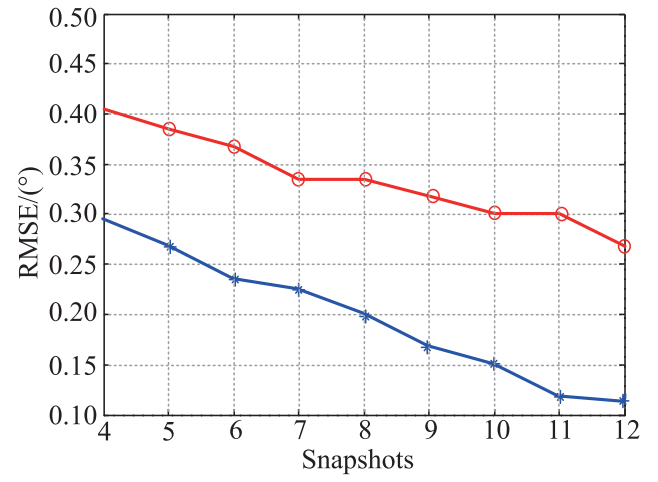

(b) RMSE

$\rightarrow$ : ITER_SPEC; $\_$: TCT.

Fig. 2 Performance versus different snapshots when $\mathrm{SNR}=10 \mathrm{~dB}$

Fig. 3 gives the performance of DOA estimation for the TCT method and the proposed method in different SNRs, where the number of the snapshots is 10. Fig. 3(a) is the detected probability, Fig. 3(b) is the RMSE of the angle estimate. Fig. 3 shows that the TCT method can get the correct estimated value of DOA when the SNR is greater than $6 \mathrm{~dB}$. However, the proposed method can obtain good estimated performance only when the SNR is $0 \mathrm{~dB}$. Thus the spectral reconstruction method is better than the TCT method with the small snapshots and the low SNR.

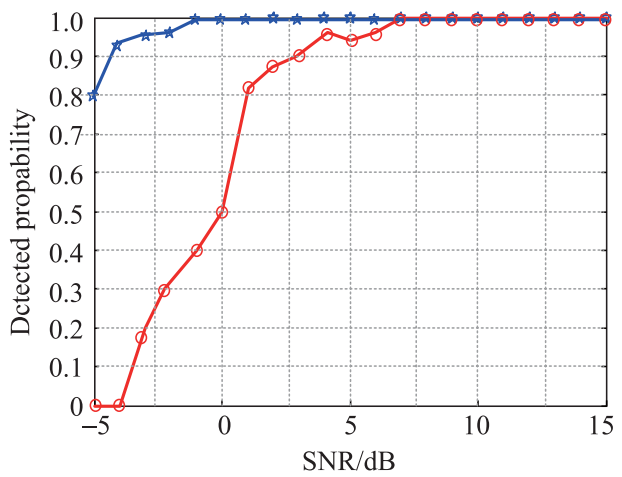

(a) Detected probability

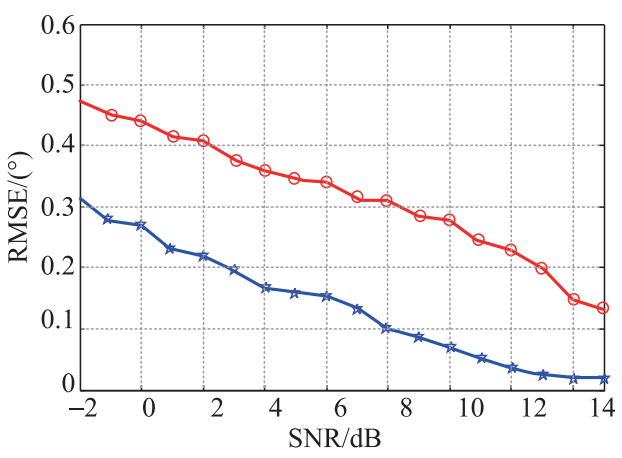

(b) RMSE

— :ITER_SPEC; $\multimap$ : TCT

Fig. 3 Performance versus different SNRs under 10 snapshots 
The above simulation results show the proposed method can obtain better estimation performance than the TCT method under the conditions of the small samples and the low SNR, the essence is that the proposed method makes full use of the deterministic transformation property of the array manifold at different frequency bins to reconstruct the covariance matrix, thus avoiding the problem of determining the signal subspace accurately of the coherent signal subspace method.

Considering a twenty-sensors uniform linear array (ULA), and two groups of coherent sources, Fig. 4 further gives the normalized minimum mean square spectrum (MMSS) in different iteration times using the proposed method, where the first group of coherent sources are placed at $-1^{\circ}$ and $3^{\circ}$, the second group of coherent sources are placed at $25^{\circ}, 30^{\circ}$ and $35^{\circ}$, the two groups of sources are independent and the SNR of sources is $5 \mathrm{~dB}$, the snapshots are 10, other simulation parameters are the same as before. Because the sequential update for the covariance matrices of all frequency bins is adopted, the proposed method can realize the full dimensional focusing for data of different frequency bins, which can deal with coherent sources.

The results show that the MMSS calculated by the proposed method can form the correct spectral peaks at the DOA of sources after three or four iterations.

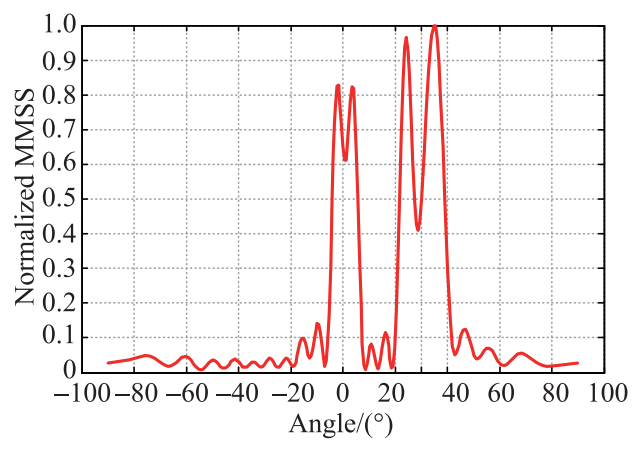

(a) The first iteration

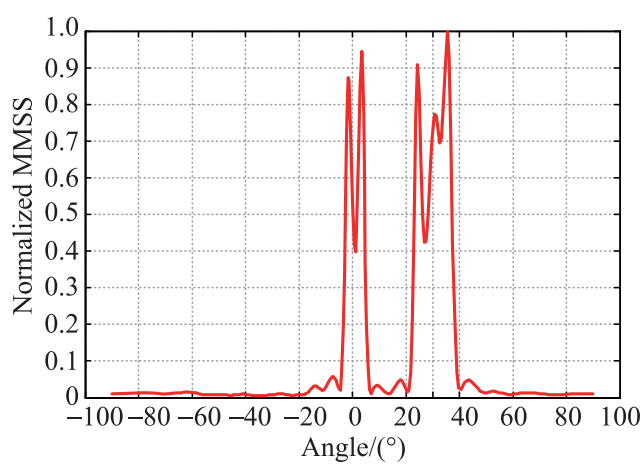

(b) The second iteration

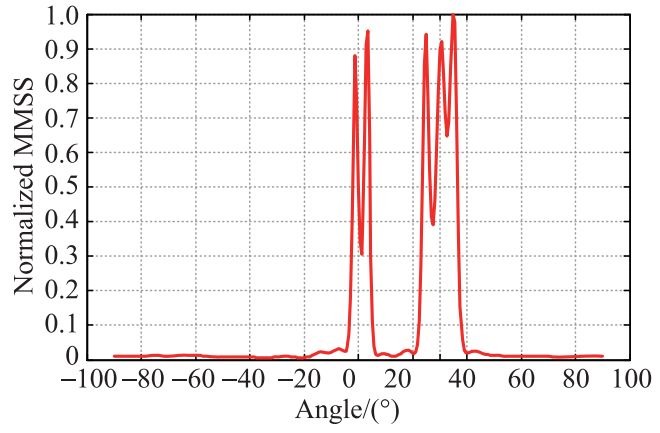

(c) The third iteration

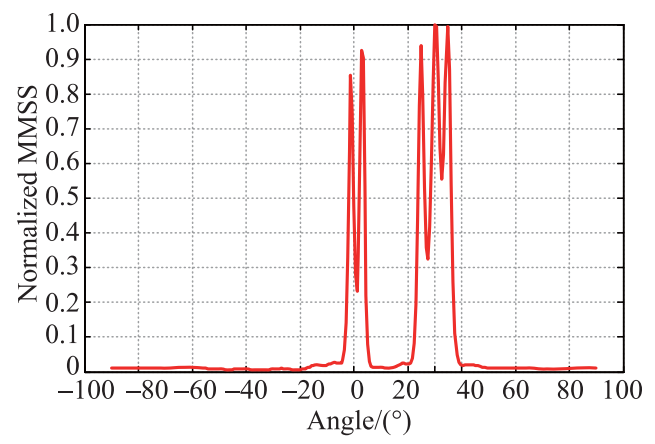

(d) The fourth iteration

Fig. 4 Variation of MMSS with different iteration times

\section{Conclusions}

This paper presents a DOA estimation method for wideband sources. In the method, the initial covariance matrix of data at the referenced frequency bin is firstly formed by the matched power spectrum of the referenced frequency bin; then the linear restrained minimum variance spectra in other frequency bins are reconstructed using the sequential iterative method; finally, the DOA of sources can be obtained by the locations of spectral peaks. Because the sequential update for the auto-correlative matrices of all frequency bins is adopted, the proposed method can realize the full dimensional focusing for data of different frequency bins, which can obtain good estimate performance with the small snapshots and the low SNR. The proposed method is also applicable to coherent sources. The experiment results indicate the proposed method has better performance of angle estimation than the TCT method with the small snapshots and the low SNR.

\section{References}

[1] K. Mahata. A subspace algorithm for wideband source localization without narrowband filtering. IEEE Trans. on Signal Processing, 2011, 59(7): 3470-3475.

[2] L. Kumar, A. Tripathy, R. M. Hegde. Robust multi-source localization over planar arrays using MUSIC-group delay spectrum. IEEE Trans. on Signal Processing, 2014, 62(117): $4627-4636$

[3] P. Jie, J. J. Zhou. Khatri-Rao subspace wideband DOA estimation based on convex optimization. Journal of Electronics \& 
Information Technology, 2013, 35(1): 81-84.

[4] M. Wax, T. J. Shan, T. Kailath. Spatio-temporal spectral analysis by eigenstructure methods. IEEE Trans. on Acoustics, Speech, Signal Processing, 1984, 32(4): 817-827.

[5] T. J. Shan, M. Wax, T. Kailath. On spatial smoothing for direction of arrival estimation of coherent signals. IEEE Trans. on Acoustics, Speech, Signal Processing, 1985, 33(4): 806-811.

[6] H. Wang, M. Kaveh. Coherent signal-subspace processing for the detection and estimation of angles of arrival of multiple wideband sources. IEEE Trans. on Acoustics, Speech, Signal Processing, 1985, 33(4): 823-831.

[7] H. Hung, M. Kaveh. Focussing matrices for coherent signalsubspace processing. IEEE Trans. on Acoustics, Speech, Signal Processing, 1988, 36(8): $1272-1281$.

[8] M. A. Doron, A. J. Weiss. On focusing matrices for wide-band array processing. IEEE Trans. on Signal Processing, 1992, 40(6): $1295-1302$.

[9] H. Hung, C. Mao. Robust coherent signal-subspace processing for directions of arrival estimation of wideband sources. IEEE Proceedings of Radar, Sonar and Navigation, 1994, 141(5): $256-262$.

[10] S. Valaee, B. Champagne, P. Kabal. Localization of wideband signals using least-squares and total least-squares approaches. IEEE Trans. on Signal Processing, 1999, 47(5): 1213 - 1222.

[11] Y. S. Yoon, L. M. Kaplan, J. H. McClellan. TOPS: new DOA estimator for wideband signals. IEEE Trans. on Signal Processing, 2006, 54(6): $1977-1989$.

[12] J. A. Luo, X. P. Zhang, Z. Wang. A new subband information fusion method for wideband DOA estimation using sparse signal representation. IEEE International Conference on Acoustics, Speech and Signal Processing, 2013: 4016-4020.

[13] P. Rocca, G. Oliveri, F. Viani, et al. Directions-of-arrival estimation through Bayesian compressive sensing strategies. IEEE Trans. on Antennas and Propagation, 2013, 61(7): $3828-$ 3838.

[14] Z. M. Liu, Z. T. Huang, Y. Y. Zhou. Direction-of-arrival estimation of wideband signals via covariance matrix sparse representation. IEEE Trans. on Signal Processing, 2011, 59(9): $4256-4270$.

[15] Z. Q. He, Z. P. Shi, L. Huang, et at. Undertetermined DOA esti- mation for wideband signals using robust sparse covariance fitting. IEEE Signal Processing Letters, 2015, 22(4): 435 - 439.

[16] S. Y. Wu, G. S. Liao, Z. W. Yang. Direction of arrival estimation of wideband signal based on particle filters. Acta Electronica Sinica, 2011, 39(6): 1353-1357.

\section{Biographies}

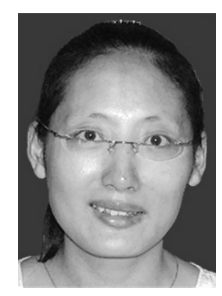

Shun He was born in 1980. She received her B.S. degree from Guilin University of Electronic Technology in 2005, Ph.D. degree in the National Laboratory of Radar Signal Processing, Xidian University in 2016. Her current research interests include adaptive array signal processing, and wideband signal processing.

E-mail: heshun1212@163.com

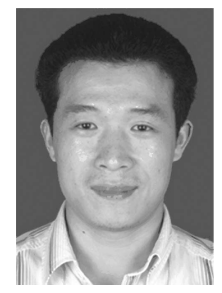

Zhiwei Yang was born in 1980. He received his M.S. and Ph.D. degrees in electrical engineering from Xidian University in 2005 and 2008, respectively. He is currently an associate professor with the National Laboratory of Radar Signal Processing, Xidian University. His current research interests include adaptive array signal processing, spacetime-polarmetric adaptive processing and design of the ground moving target indication algorithms for the space-time SAR/GMTI systems.

E-mail: zwyang@mail.xidian.edu.cn

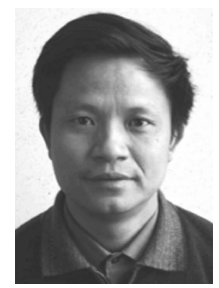

Guisheng Liao was born in 1964. He received his M.S. and Ph.D. degrees from Xidian University in 1990 and 1992, respectively. He is currently a professor with the National Laboratory of Radar Signal Processing, Xidian University. His current interests include adaptive array signal processing, ground moving target indication and distributed satellite SAR/GMTI system design.

E-mail: gsliao@xidian.edu.cn 